\title{
Single envelope equation modelling of frequency comb generation in quadratic and cubic nonlinear resonators
}

\author{
Tobias Hansson, ${ }^{1}$ François Leo, ${ }^{2 *}$ Miro Erkintalo, ${ }^{1}$ Stéphane Coen, ${ }^{2}$ Iolanda Ricciardi, ${ }^{3}$ \\ Maurizio De Rosa, ${ }^{3}$ and Stefan Wabnitz ${ }^{3,4}$ \\ ${ }^{1}$ Department of Applied Physics, Chalmers University of Technology, SE-41296 Göteborg, Sweden \\ ${ }^{2}$ The Dodd-Walls Centre for Photonic and Quantum Technologies, Department of Physics, The University of \\ Auckland, Auckland 1142, New Zealand \\ ${ }^{3}$ CNR-INO, Istituto Nazionale di Ottica, Via Campi Flegrei 34, 80078 Pozzuoli (NA), Italy \\ ${ }^{4}$ Dipartimento di Ingegneria dell'Informazione, Università di Brescia, via Branze 38, 25123 Brescia, Italy \\ ${ }^{*}$ Corresponding author: f.leo@auckland.ac.nz.
}

\begin{abstract}
We introduce the single envelope equation for the numerical modelling of ultra-broadband optical frequency comb generation via phase-matched parametric mixing in a resonator including both quadratic and cubic nonlinearities.
\end{abstract}

OCIS codes: (190.4410) Nonlinear optics, parametric processes, (190.2620) Harmonic generation and mixing

There is currently much interest in developing microscale, low pump power light sources based on nonlinear parametric frequency conversion. Low threshold parametric mixing is enabled by the high field confinement in quasiphase-matched (QPM) whispering gallery modes (WGMs) of optical microresonators [1]. By using radially poled resonators, low power and highly tunable optical parametric oscillation (OPO) has been demonstrated $[2,3]$. Very interestingly, recent experiments have demonstrated that macro-cavity second-harmonic generation (SHG) may also lead to optical frequency comb (OFC) generation $[4,5]$.

Nonlinear microresonator based sources of OFCs are a promising alternative to mode-locked lasers for several applications, ranging from high-precision metrology, coherent communications to biomedical and environmental spectroscopy [6]. We recently developed a narrowband theoretical description of OFC generation by cavity SHG, based on separate time domain evolution equations for coupled OFCs, centered around the fundamental frequency and the second harmonic, respectively [7].

In this work, we present an ultra-broadband model of OFC generation in nonlinear cavities with both quadratic and cubic nonlinear response, based on the single envelope equation (SEE) [8]. The SEE model permits to describe situations where the different parametric combs start to overlap, or when mixing between the primary combs and higher-order harmonic and phase matching processes occurs, since all processes can be modeled at once with a single equation. We apply here the SEE approach to OFC generation in a doubly resonant, radially poled lithium niobate microring resonator. OFC dynamics is modeled by combining the SEE with proper boundary conditions [9], viz.

$$
\begin{aligned}
& \mathscr{F}\left[A^{m+1}(t, 0)\right]=\sqrt{\hat{\theta}(\Omega)} \mathscr{F}\left[A_{i n}\right]+\sqrt{1-\hat{\theta}(\Omega)} e^{i \phi_{0}} \mathscr{F}\left[A^{m}(t, L)\right], \\
& {\left[\partial_{z}-D+\frac{\alpha_{d}}{2}\right] A^{m}(t, z)=i \rho_{0}\left(1+i \tau_{s h} \frac{\partial}{\partial t}\right) p_{N L}\left(t, z, A^{m}\right),}
\end{aligned}
$$

where Eq.(1) is written in the Fourier domain, $\Omega=\omega-\omega_{0}, \omega_{0}$ is a reference frequency, and $\mathscr{F}[\cdot]=\int_{-\infty}^{\infty} \cdot e^{i \Omega \tau} \mathrm{d} \tau$ denotes Fourier transformation. Here the independent variables are the evolution variable $z$, which is the longitudinal coordinate measured along the waveguide, and $t$ which is the (ordinary) time. Eq.(1) is the boundary condition that determines the intracavity field $A^{m+1}(t, z=0)$ at the input of roundtrip $m+1$ in terms of the field from the end of the previous roundtrip $A^{m}(t, z=L)$ and the pump field $A_{i n}$. The path length of the resonator is assumed to be equal to $L$.

Additionally, $\hat{\theta}(\Omega)$ is the frequency dependent transmission coefficient between the resonator and the bus waveguide, and $\delta_{0}=2 \pi l-\phi_{0} \approx\left(\omega_{R}-\omega_{0}\right) t_{R}$ is the linear phase-shift, with $\delta_{0}$ the detuning from the cavity resonance $\omega_{R}$ (assumed to correspond to the longitudinal mode number $l$ ) which is the closest to the pump frequency $\omega_{0}$, and $t_{R}$ is the cavity round-trip time. Moreover, $\rho_{0}=\omega_{0} / 2 n_{0} c \varepsilon_{0}, \varepsilon_{0}$ is the vacuum permittivity, $n_{0}=n\left(\omega_{0}\right), \omega_{0}$ is the pump frequency, $n$ is the linear refractive index, and the shock coefficient $\tau_{s h}=1 / \omega_{0}$, and $\alpha_{d}$ is the distributed linear loss 
coefficient. $D$ is the usual group-velocity dispersion (GVD) operator. In Eq.(2) the nonlinear polarization $p_{N L}$ is given by the sum of the quadratic and cubic contributions

$p_{N L}^{(2)}(t, z, A)=\frac{\varepsilon_{0} \chi^{(2)}}{2}\left[2|A|^{2} \exp (i \psi(t, z))+A^{2} \exp (-i \psi(t, z))\right], p_{N L}^{(3)}(t, z, A)=\frac{\varepsilon_{0} \chi^{(3)}}{4}\left[3|A|^{2} A+A^{3} \exp (-2 i \psi(t, z))\right]$,

where $|A|^{2}$ only contains positive frequency $\omega_{0} \geq 0$ components, $\psi(t, z)=\omega_{0} t-\left(\beta_{0}-\beta_{1} \omega_{0}\right) z, \beta_{0}=\beta\left(\omega_{0}\right)$, and $\beta_{1}=\beta^{\prime}\left(\omega_{0}\right)$.
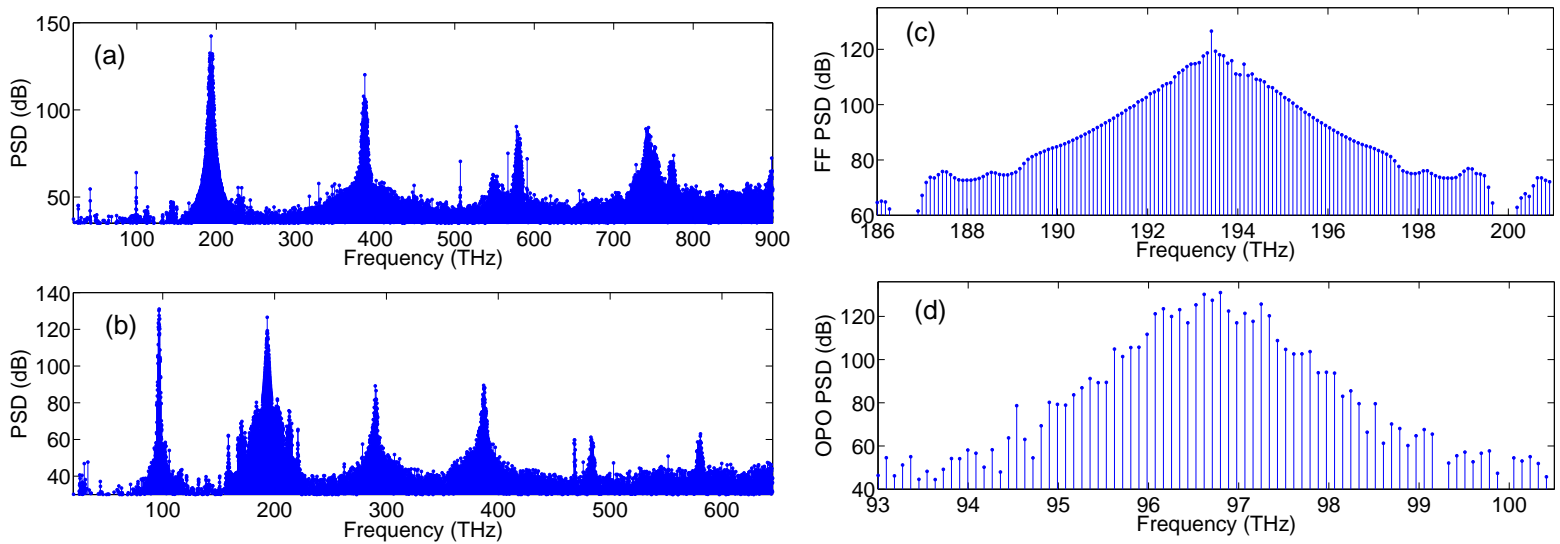

Fig. 1. Ultra-broadband OFC in quadratic and cubic micro-resonator. Power spectral density (PSD) from intra-cavity (a) SHG and (b) OPO; Output OFC spectra around (c) the fundamental (FF) and (d) the degenerate OPO frequencies.

We numerically integrate Eq.(1) and (2) for two different cases corresponding to a quasi phase matched SHG and OPO process respectively. In both cases, the pump is at $1550 \mathrm{~nm}(193 \mathrm{THz})$. In Fig. 1 (a) we illustrate the spectrum of OFCs generated in a doubly-resonant, radially poled lithium niobate microring resonator with QPM of SHG, with the $\mathrm{CW}$ fundamental pump power $P_{i n}=20 \mathrm{~mW}$ at $1550 \mathrm{~nm}$. In the simulations, we considered a spectral grid of a single free-spectral range and a quantum noise seed. Although intra-cavity SHG leads to the generation of two main combs around the fundamental and the second harmonic, additional combs also appear at the third and fourth harmonic. Fig. 1 (b) shows the output comb spectrum when the QPM leads to degenerate OPO in the mid-infrared (MIR). Details of the combs generated around the fundamental and the OPO wavelengths are given in Figs. 1 (c) and (d). Quite interestingly, the triangular shape of the fundamental spectral profile is associated with a single, phase locked hyperbolic secant solitary pulse per round-trip.

In summary, we have shown that the SEE approach permits to numerically simulate with a single equation both quadratic and cubic nonlinear processes leading to complex ultra-broadband coherent OFCs ranging from the the visible to the MIR region.

\section{References}

1. V. S. Ilchenko, A. B. Matsko, A. A. Savchenkov, and L. Maleki, J. Opt. Soc. Am. B 20, 1304 (2003).

2. A. Savchenkov, A. B. Matsko, M. Mohageg, D. V. Strekalov, and L. Maleki, Opt. Lett. 32, 157 (2007).

3. T. Beckmann, H. Linnenbank, H. Steigerwald, B. Sturman, D. Haertle, and I. Breuning, Phys. Rev. Lett. 106, 143903 (2011).

4. V. Ulvila, C. R. Phillips, L. Halonen, and M. Vainio, Opt. Lett. 38, 4281 (2013).

5. I. Ricciardi, S. Mosca, M. Parisi, P. Maddaloni, L. Santamaria, P. De Natale, and M. De Rosa, Phys. Rev. A 91, 063839 (2015).

6. T. J. Kippenberg, R. Holzwarth, and S. A. Diddams, Science 332, 555 (2011).

7. F. Leo, T. Hansson, I. Ricciardi, M. De Rosa, S. Coen, S. Wabnitz, and M. Erkintalo, Phys. Rev. Lett. 116, 033901 (2016).

8. F. Baronio, M. Conforti, C. De Angelis, D. Modotto, S. Wabnitz, M. Andreana, A. Tonello, P. Leproux, and V. Couderc, Opt. Fiber Techn. 18, 283 (2012).

9. M. Haelterman, S. Trillo, and S. Wabnitz, Opt. Commun. 91, 401 (1992). 